
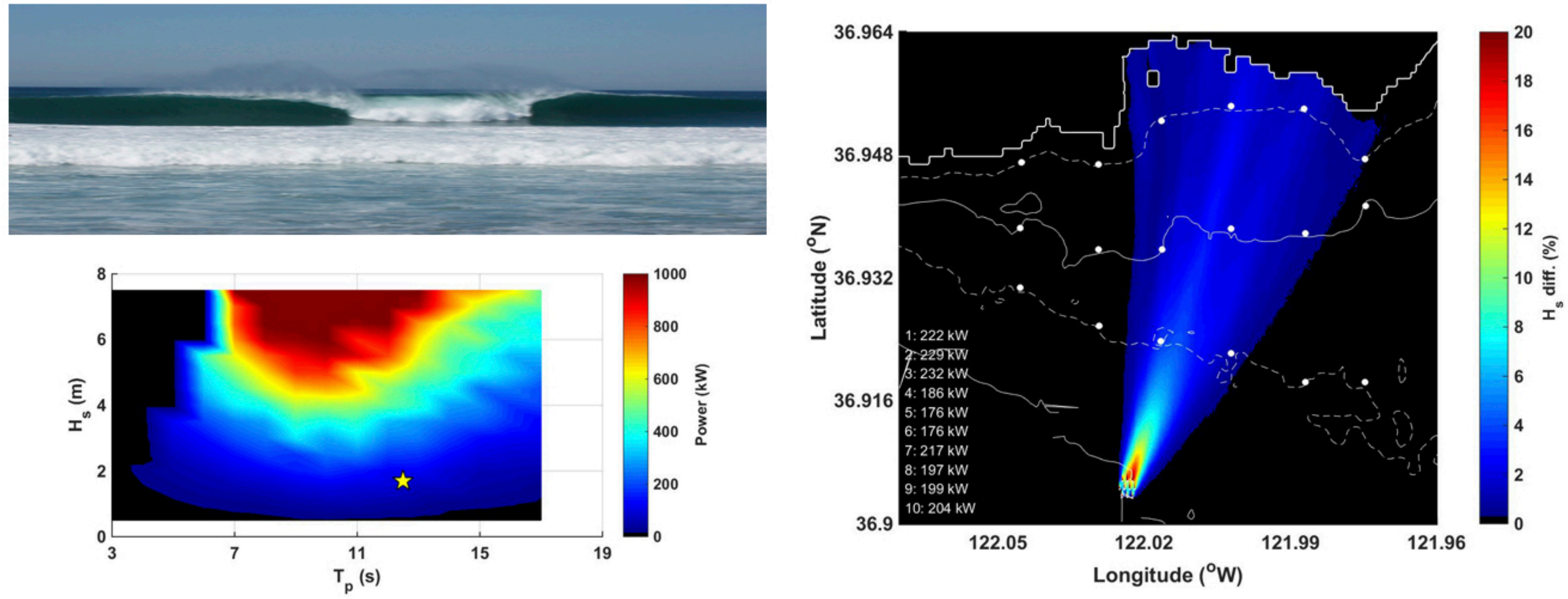


\section{Numerical modeling of the effects of wave energy converter characteristics on \\ nearshore wave conditions}

\section{Abstract}

15 Modeled nearshore wave propagation was investigated downstream of simulated wave energy

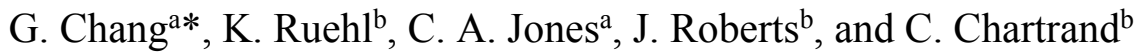

antegral Consulting Inc., 200 Washington Street, Suite 101, Santa Cruz, CA 95060 USA;

Phone: 805.967.5640; Email: gchang@integral-corp.com and cjones@integral-corp.com

bSandia National Laboratories, P.O. Box 5800, Albuquerque, NM 87185 USA;

Email:kmruehl@sandia.gov,jdrober@sandia.gov, and ccchart@sandia.gov

*Corresponding author 
24 incident wave height. Other WEC types resulted in less than $15 \%$ differences in modeled wave

25 height with and without WECs, with lesser influence for WECs less than $10 \mathrm{~m}$ in diameter.

26 Wave directions and periods were largely insensitive to changes in parameters. However,

27 additional model parameterization and analysis are required to fully explore the model sensitivity

28 of peak wave period and mean wave direction to the varying of the parameters.

30 Keywords: wave energy converter (WEC); wave modeling; model sensitivity; Simulating WAves

31 Nearshore (SWAN); nearshore wave propagation

\section{1. Introduction}

35 In order to effectively convert wave energy into commercial-scale onshore electrical power, 36 arrays of multiple wave energy converter (WEC) devices are necessary. The deployment of

37 WEC arrays will likely begin small (pilot-scale or $\sim 10$ devices) but could feasibly number in the

38 hundreds of individual devices at commercial-scale. As the industry progresses from pilot- to

39 commercial-scale, an understanding of the effects of WEC arrays leeward of the deployment site

40 and on the nearshore environment will become increasingly important. WEC arrays have the

41 potential to alter nearshore wave propagation and circulation patterns and possibly modify

42 sediment transport patterns (e.g., Gonzalez-Santamaria et al., 2013), which could have

43 detrimental effects on ecological processes and local socioeconomic services. To help accelerate

44 the realization of commercial-scale wave power, it is necessary to evaluate the potential

45 environmental effects of WEC arrays and inform environmental assessments associated with the

46 regulatory process (e.g., Copping et al., 2014; Leeney et al., 2014; Tiron et al., 2015). 
48 At present, due to the lack of deployed WEC farms, direct measurements of the effects of WEC

49 arrays on nearshore wave propagation are not available. Wave model simulations however, can

50 provide the groundwork for completing such environmental assessments by investigating the

51 sensitivity of predictive model results to differing WEC characteristics over anticipated wave

52 conditions. The understanding developed here will allow investigators to conduct predictive

53 environmental assessments with increased confidence and reduced uncertainty in future phases

54 of WEC development.

1.1 Background

58 Baseline versions of spectral wave models, such as TOMAWAC (TOMAWACE, 2014) and

59 SWAN (Simulating WAves Nearshore) (Booij et al., 1999; Ris et al., 1999) do not have the

60 inherent capabilities needed for modeling far-field effects of arrays of WECs. These codes

61 effectively model a WEC as an obstacle with a constant, user-specified transmission coefficient

62 (Goda, 1967; d'Angremond et al., 1996) applied across the entire frequency spectrum.

63 Transmission coefficients determine the amount of wave energy that is absorbed and

64 subsequently allowed to transmit past the obstacles, or WECs.

65

66 In a study presented by Millar et al. (2007), potential WEC farm effects on shoreline change

67 were modeled at the Wave Hub site using the native SWAN model and transmission coefficients

68 set at $0 \%, 40 \%, 70 \%$, and $90 \%$ (corresponding to $100 \%, 60 \%, 30 \%$, and $10 \%$ wave energy

69 absorption). Millar et al. (2007) concluded that wave heights inshore of WECs decrease linearly 
70 with increasing wave energy transmission. Bento et al. (2014) also simulated WECs as SWAN

71 obstacles with the same transmission coefficients at three different incoming wave directions

72 along the Portuguese coast during two different seasons. They similarly found decreases in

73 significant wave and swell height with increased energy absorption immediately in the lee of the

74 simulated WEC farm.

76 Several other studies by Rusu and Guedes Soares (e.g., 2008, 2009, and 2013) evaluated WEC

77 and WEC farm effects on the Portuguese coast and neighboring archipelagos. Iglesias and

78 Carballo (2014) determined impact indicators to describe the influence of WEC farm distance to

79 shore on nearshore wave characteristics using constant transmission coefficients determined

80 from laboratory studies. Wave farm configuration sensitivity analysis was performed for the

81 Pelamis WEC by Palha et al. (2010). Chang et al. (2014) evaluated the sensitivity of the native

82 SWAN model to a variety of model parameters with and without a WEC array (transmission

83 coefficient, frequency spreading, directional spreading, and WEC device spacing within the

84 array) and concluded that changes in significant wave height in the lee of a simulated WEC array

85 are most sensitive to wave energy transmission.

87 While these studies provided insight on wave propagation in the presence of an array of

88 obstacles, they did not take into account WEC device-specific characteristics in the specification

89 of the SWAN model's obstacle transmission coefficients. For example, a later review of the

90 study by Millar et al. (2007) determined that the application of constant obstacle transmission

91 coefficients to model WECs was not well understood and there was not sufficient guidance on

92 how to appropriately account for WEC power performance (ASR Limited, 2007). This directly 
93 motivated the work accomplished by Smith et al. (2012), who modified the SWAN code to

94 account for the frequency- and directionally-dependent power absorption of WECs. With

95 Smith's modifications to SWAN, WEC power performance can be modeled by user-defined

96 frequency- and directionally-depended power transfer functions. Using this model, the effects of

97 deploying wave farms at the Wave Hub site were re-assessed (Smith et al., 2012).

99 The development of the SNL-SWAN (Sandia National Laboratories - SWAN) code builds upon

100 the work performed by Smith et al. (2012) by further modifying the native SWAN code to allow

101 for direct importation of WEC power performance data in the form of relative capture width

102 (RCW) curves, or power matrices. RCW curves and power matrices are the current industry

103 standard practice for defining WEC power production (e.g., Hagerman and Bedard, 2003) (RCW

104 curves are analogous to turbine power curves in the wind industry). The incorporation of RCW

105 curves or power matrices removes any uncertainties related to arbitrarily chosen transmission

106 coefficient values. Rather, the transmission coefficients (or WEC power absorption) are

107 calculated directly by SNL-SWAN based on user-defined WEC power performance data. This

108 approach has been verified by comparison to other codes and has undergone preliminary

109 validation by comparison to experimental wave tank data (Ruehl et al., 2013; Porter et al., 2014).

110

111 1.3 Objectives

112

113 The present study incorporates SNL-SWAN, a modified version of an industry standard wave

114 modeling tool, SWAN to simulate wave propagation through a hypothetical WEC array

115 deployment site on the California coast. The primary objective of the SNL-SWAN sensitivity 
116 study is to investigate the potential effects of a range of WEC devices on leeward and nearshore

117 wave propagation. To accomplish this goal, the following tasks are undertaken:

118 Evaluate the modified wave propagation model, SNL-SWAN, which allows the

119 incorporation of device-specific WEC characteristics.

120 - Perform model sensitivity analysis using SNL-SWAN to examine the effects of WEC

121 characteristics (WEC device type and size, number of WECs, and WEC device spacing

122 within the WEC array) on near-field and far-field wave conditions in the lee of the WEC

123 devices.

125 2. Methods

126

127 2.1 SNL-SWAN

128

129 SNL-SWAN was developed to more accurately evaluate WEC farm effects on wave propagation

130 by incorporating a WEC module that accounts for device-specific WEC power performance.

131 Based on the user specified power performance, SNL-SWAN calculates transmission

132 coefficients that are associated with a WEC's power performance. Several methods of

133 determining the transmission coefficient are employed in Version 1.0 of SNL-SWAN. The five

134 methods are employed through switches (specified in the SNL-SWAN input file) in the SNL-

135 SWAN WEC module, where:

137 Switch 0) SNL-SWAN uses the standard SWAN obstacle treatment (Delft University of 138 Technology, 1993). The transmission coefficient value, $K_{t}$, is a constant value entered into the 
139 SWAN input file and applied across all wave frequencies. The transmission coefficient

140 represents the ratio of wave heights incident to and in the lee of the obstacle (or WEC) (Eq. (1)).

$$
K_{t}=\frac{H_{\text {lee }}}{H_{\text {incident }}}
$$

142 Switch 1) SNL-SWAN computes the transmission coefficient from a user-supplied WEC power

143 matrix (Table 1 shows an example power matrix for a particular WEC). A power ratio is then

144 calculated at the peak wave period based on the absorbed wave power from the WEC power

145 matrix (supplied by the user) and the incident wave power (determined from SNL-SWAN). The

146 transmission coefficient used by SNL-SWAN is calculated based on this power ratio at the peak

147 wave period, as shown in Eq. (2), and is applied as a constant value across all wave frequencies.

$$
K_{t}^{2}=\frac{P_{\text {Lee }}}{P_{\text {Incident }}}=\frac{P_{\text {Incident }}-P_{\text {Absorbed }}}{P_{\text {Incident }}}=1-\frac{P_{\text {Absorbed }}}{P_{\text {Incident }}}
$$

149 Switch 2) SNL-SWAN computes the transmission coefficient from a user-supplied WEC RCW

150 curve. The transmission coefficient used by SNL-SWAN is calculated based on the RCW value

151 from the curve given the peak incident wave period, as shown in Eq. (3), and is applied as a

152 constant value across all wave frequencies.

$$
K_{t}^{2}=1-\frac{P_{\text {Absorbed }}}{P_{\text {Incident }}}=1-R C W
$$

154 Switch 3) SNL-SWAN switch 3 is an extension of switch 1, where distinct transmission

155 coefficients are applied to each binned wave frequency based on the WEC power matrix. This

156 results in varying power absorption for different wave frequencies as $K_{t}^{2}$ is a function of wave

157 frequency. 
159 Switch 4) SNL-SWAN switch 4 is an extension of switch 2, where the RCW curve is sampled

160 independently for each binned wave frequency, resulting in a frequency dependent obstacle

161 transmission coefficient. Please refer to the SNL-SWAN user's manual for more information on

162 the SNL-SWAN WEC module (Ruehl et al., 2014).

163

164 Table 1. Power matrix computed for a floating two-body heaving converter (select wave periods 165 and heights shown) (Babarit et al. 2012).

\begin{tabular}{|c|c|c|c|c|c|c|c|c|c|}
\hline & \multicolumn{8}{|c|}{$T_{p}(s)$} \\
\hline & & 3 & 5 & 7 & 9 & 11 & 13 & 15 & 17 \\
\hline \multirow{7}{*}{ 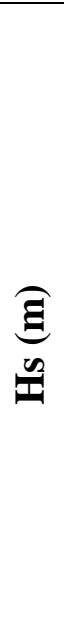 } & 1 & 2.38 & 11.41 & 24.59 & 43.58 & 53.08 & 34.42 & 19.86 & 11.78 \\
\hline & 2 & 0 & 45.28 & 100.01 & 153.17 & 150.81 & 126.08 & 60.69 & 48.03 \\
\hline & 3 & 0 & 96.17 & 204.65 & 357.25 & 352.72 & 248.15 & 136.67 & 112.52 \\
\hline & 4 & 0 & 0 & 366.25 & 550.98 & 530.82 & 419.89 & 268.44 & 193.75 \\
\hline & 5 & 0 & 0 & 514.29 & 824.37 & 617.58 & 512.41 & 384.04 & 257.61 \\
\hline & 6 & 0 & 0 & 710.80 & 973.55 & 838.26 & 648.39 & 501.78 & 388.41 \\
\hline & 7 & 0 & 0 & 781.16 & 1000 & 1000 & 959.05 & 573.85 & 449.84 \\
\hline
\end{tabular}

168 In this paper, all five switches were implemented for a hypothetical WEC array in Monterey

169 Bay, CA. Although it is not an ideal WEC farm location with respect to its wave climatology, the

170 site was chosen based on available wave model validation data as well as previous wave model

171 assessments conducted in the region. The sensitivity analysis was performed for the power 
172 matrices of a variety of existing WEC devices based on the numerical benchmarking paper by

173 Babarit et al. (2012).

174

1752.2 Wave Model Validation: Monterey Bay, CA

176 SNL-SWAN wave propagation calculations defer to the native SWAN code for cases without

177 obstacles. Therefore, initial site-specific wave model validation was accomplished using the

178 native SWAN model. Deep-water waves were propagated from offshore to shallow water for a

179 study site in Monterey Bay and coastal Santa Cruz, CA. Several local National Oceanic and

180 Atmospheric Administration National Data Buoy Center (NOAA NDBC) buoys provided

181 measurements of wave heights, wave periods, wave directions, wind speeds, and wind directions

182 at Monterey Bay buoy locations dating as far back as 1987. Data from NDBC buoy 46042

$183\left(36^{\circ} 47^{\prime} 29^{\prime \prime} \mathrm{N}, 122^{\circ} 27^{\prime} 6^{\prime \prime} \mathrm{W} ; 2098 \mathrm{~m}\right.$ water depth) were used to derive Monterey Bay domain

184 boundary conditions. Data from NDBC buoy $46236\left(36^{\circ} 45^{\prime} 39^{\prime \prime} \mathrm{N}, 121^{\circ} 56^{\prime} 48^{\prime \prime} \mathrm{W} ; 145 \mathrm{~m}\right.$ water

185 depth) were used to validate the Monterey Bay SWAN model predictions for significant wave

186 height $\left(\mathrm{H}_{\mathrm{s}}\right)$, peak wave period $\left(\mathrm{T}_{\mathrm{p}}\right)$, and mean wave direction (MWD). Additionally, a Datawell

187 DWR-G4 wave buoy was deployed in 5 to $6 \mathrm{~m}$ water depth near the Santa Cruz Harbor, Santa

188 Cruz, $\mathrm{CA}\left(36^{\circ} 57^{\prime} 35^{\prime \prime} \mathrm{N}, 121^{\circ} 59^{\prime} 56^{\prime \prime} \mathrm{W}\right)$ for nearshore wave model validation. The model

189 performance statistics computed for the Monterey Bay and Santa Cruz, CA SWAN model

190 studies showed good agreement between modeled to measured data (Table 2). The wave model

191 and validation are further discussed in Chang et al. (2010, unpublished).

192

193 
194 Table 2. Native SWAN model error statistics calculated for the Monterey Bay and Santa Cruz,

195 CA wave model validation studies.

\begin{tabular}{c|ccc|ccc}
\hline & \multicolumn{3}{|c|}{ Monterey Bay } & \multicolumn{3}{c}{ Santa Cruz } \\
\hline Wave Parameter & RMSE & SI & ME & RMSE & SI & ME \\
& & & & & & \\
\hline $\mathrm{H}_{\mathrm{s}}$ & 0.29 & 0.17 & -0.06 & 0.19 & 0.22 & 0.04 \\
\hline $\mathrm{T}_{\mathrm{p}}$ & 2.78 & 0.26 & -0.37 & 1.20 & 0.09 & 0.37 \\
\hline MWD & 21.6 & 0.08 & 6.34 & 6.92 & 0.03 & -1.53 \\
\hline
\end{tabular}

198 All SWAN and SNL-SWAN model validation, as well as verification, evaluation, and sensitivity 199 analysis runs (see Sections 2.3 and 2.4) employed nautical coordinates in a spherical grid. The 200 computational grids were uniform and rectangular. Monterey Bay high resolution digital 201 bathymetric data were obtained from the NOAA National Geophysical Data Center (NGDC; 202 https://www.ngdc.noaa.gov/mgg/bathymetry/hydro.html) and Santa Cruz model domain bottom 203 bathymetry was obtained from high resolution single-beam survey data obtained in November 2042009 (Chang et al., 2010, unpublished). The number of directional meshes was specified as 40 205 equally spaced subdivisions of a $360^{\circ}$ circle (every $9^{\circ}$ ). The frequency resolution was 24 206 logarithmically spaced frequencies between 0.042 and $1 \mathrm{~Hz}$. SWAN and SNL-SWAN were 207 commanded to utilize the JONSWAP spectrum with default parameters. SWAN was run in first208 generation mode with default diffusion and Collins (1972) bottom friction coefficient, wave 209 breaking and triad wave-wave interactions turned on, and diffraction turned off. 
212 SNL-SWAN was evaluated for functionality and comparability to the native SWAN code by

213 using a two-nested model domain in Monterey Bay and Santa Cruz, CA, similar to that presented

214 by Chang et al. (2014) (Figure 1). The coarse-grid (herein referred to as the Monterey Bay model

215 domain) resolution was $0.001^{\circ}$ in latitude and longitude. The model was run as a stationary

216 model, i.e. meteorological and hydrodynamic conditions at the offshore boundaries were kept

217 constant. Directional wave energy spectra conditions were exported from the coarse resolution

218 model and used as boundary conditions for the nested, fine resolution model (herein referred to

219 as the Santa Cruz model domain).

221 The grid resolution of the nested Santa Cruz model domain computational grid was matched to

222 the size of the modeled WEC device type. For SNL-SWAN model verification purposes, the

223 device size and type chosen was a 20-m floating two-body heaving converter (F-2HB; Babarit,

224 2012). The Santa Cruz model grid size was therefore $0.0002^{\circ}$ in latitude and longitude. The wave

225 spectrum boundary conditions were applied along the offshore boundaries of the Santa Cruz

226 SNL-SWAN model domain. The nested grid model was also implemented as a stationary model.

228 SNL-SWAN was operated using switch 0 and compared to results from the native SWAN model

229 to ensure model integrity. The SNL-SWAN and native SWAN model initial wave conditions

230 were: $\mathrm{H}_{\mathrm{s}}=1.5 \mathrm{~m}, \mathrm{~T}_{\mathrm{p}}=12.5 \mathrm{~s}, \mathrm{MWD}=205^{\circ}$, frequency distribution spread of 3.3, and directional

231 distribution spread of 25 . The model runs incorporated an array of 10 WEC devices with $86 \%$

232 transmission $\left(K_{t}=0.86\right)$, zero wave energy reflection allowed, centered on the $40 \mathrm{~m}$ depth

233 contour. The WEC device array was arranged in a diamond-shape as a representative

234 configuration (Figure 1). WEC devices were simulated in the model with 6-diameter (center-to- 
235 center) spacing between devices. Devices were equally spaced in all directions. Again, the

236 simulated WEC device type was a floating two-body heaving converter with $20 \mathrm{~m}$ diameter. The

237 model results were evaluated at six shoreline locations along the Santa Cruz coast on the $10 \mathrm{~m}$,

$23820 \mathrm{~m}$, and $30 \mathrm{~m}$ depth contours (Figure 1). As expected, SNL-SWAN Switch 0 and native

239 SWAN runs yielded identical results, i.e. SNL-SWAN modifications did not affect the

240 functionality or integrity of SWAN (Figure 2).

242 2.4 SNL-SWAN Model Evaluation

243 Following general SNL-SWAN model validation, SNL-SWAN switches 1, 2, 3, and 4 were

244 evaluated for the conditions described in 2.1.2 SNL-SWAN Model Verification except without a

245 user-specified transmission coefficient. Results were evaluated for the 18 output locations shown

246 in Figure 1. Results from all four SNL-SWAN switches were identical to each other at the

247 western and easternmost output locations (location numbers 1 through 6 and 16 through 18;

248 Figure 2). The maximum difference in simulated wave height between SNL-SWAN switch 1 and

2493 compared to switch 2 and 4 was $0.4 \mathrm{~cm}$ at the location number 7 , which is directly in the lee of

250 the WEC array with an incoming MWD of $205^{\circ}$. Differences in modeled wave height were 0.2

$251 \mathrm{~cm}$ or less at output location numbers 8 through 12; switches 1 and 3 simulated wave heights

252 were consistently 0.1 to $0.2 \mathrm{~cm}$ higher than those modeled using SNL-SWAN switches 2 and 4 .

253 These differences are likely due to the data interpolation necessary for computing the RCW

254 curve used in switches 2 and 4.

255

256 Results from employing a frequency dependent WEC transmission coefficient are minimal for 257 this particular simulation. Switch 2 and switch 4 results are identical. Only a $0.1 \mathrm{~cm}$ difference in 
258 wave height is observed at output location number 11 when comparing results from switch 1 and

259 switch 3, with slightly less power absorbed (i.e. higher wave height) when using a frequency

260 dependent transmission coefficient. Although no field validation is available for model runs with

261 simulated WECs, SNL-SWAN switches 3 and 4 are assumed to provide the most accurate

262 mechanistic depiction of wave and WEC interaction(s) (e.g., Smith et al., 2012).

264 3. SNL-SWAN Sensitivity Analysis

Model sensitivity analysis was performed using SNL-SWAN in order to understand model

267 behavior in the vicinity of a variety of different WEC devices and sizes, WEC spacing within an

268 array, and number of WECs in an array. Potential alterations to the nearshore wave climate in

269 proximity to the Santa Cruz, CA shoreline due to WECs were explored by simulating eight

270 different WEC device types with seven different diameters (Table 3) arranged in diamond-

271 shaped arrays of 10, 50, or 100 WEC devices that were equally spaced 4, 6, or 8 diameters apart,

272 center-to-center. Because SNL-SWAN is an energy propagation model and the sensitivity

273 analysis was performed to investigate the extraction of energy from incident wave spectra

274 independent of depth, all WECs were simulated as obstacles extending throughout the water

275 column. All model runs were conducted with the WEC array centered on the $40 \mathrm{~m}$ depth contour,

276 as shown in Figure 1. The power matrix for each of the eight devices was computed following

277 Babarit et al. (2012) (Figure 3). See Appendix A for a table of notations.

279 A total of 288 SNL-SWAN model runs were conducted (72 for each of SNL-SWAN switch 1, 2,

2803 , and 4). Results were compared to seven model runs conducted with no obstacles. The seven

281 runs with no WECs represented each of the seven different device sizes. The initial wave 
282 conditions were determined from statistical analysis of NDBC buoy 46042 (Monterey Bay, CA)

283 wave climatology records, where median values are: $\mathrm{H}_{\mathrm{s}}=1.7 \mathrm{~m}$ and $\mathrm{T}_{\mathrm{p}}=12.5 \mathrm{~s}$. Although the

284 median wave direction computed from NDBC buoy 46042 wave records is $299^{\circ}$, representative

285 offshore wave conditions were selected based on its potential to alter nearshore Santa Cruz, CA

286 wave properties. Therefore, the MWD chosen was $205^{\circ}$ such that wave shadowing effects by

287 land are reduced. The initial wave conditions were held constant for all model runs. SNL-SWAN

288 model parameters were selected based on SNL-SWAN model evaluation results: frequency

$289 \operatorname{spread}=3.3$, directional spread $=25$, directional resolution $=9^{\circ}$, zero wave energy reflection

290 allowed, and no diffraction.

292 Table 3. WEC device types and associated diameters (maximum of length and width; from

293 Babarit et al., 2012) simulated for SNL-SWAN model sensitivity analysis.

\begin{tabular}{llc}
\hline \multicolumn{1}{c}{ WEC type } & Abbreviation & Diameter (m) \\
\hline Small bottom-referenced heaving buoy & Bref-HB & $5^{*}$ \\
\hline Bottom-fixed heave-buoy array & B-HBA & 5 \\
\hline Bottom-referenced submerged heave-buoy ${ }^{\#}$ & Bref-SHB & 7 \\
\hline Floating heave-buoy array\% & F-HBA & 8 \\
\hline Floating three-body oscillating flap device & F3 OF & 9.5 \\
\hline Floating two-body heaving converter & F-2HB & 20 \\
\hline Bottom-fixed oscillating flap & B-OF & 50 \\
\hline Floating oscillating water column & F-OWC \\
\hline * The Bref-HB published size is 3 m; however it was specified as 5 m in SNL-SWAN model \\
runs due to limitations on computational grid size.
\end{tabular}


$296 \quad$ BB-HBA and Bref-SHB were modeled as obstacles that extended throughout the water column 297 although both are devices without surface expressions.

$298 \%$ F-HBA is a multi-body WEC, composed of any number of heaving buoys connected to a 299 submerged structure. The actual dimension of F-HBA is dependent on the number of heaving 300 buoys within its body. It was therefore modeled as a singular 8-m diameter obstacle.

302 3.1. Model Set-Up

303

304 Two or three SNL-SWAN model grids were nested, depending on WEC diameter, to predict the 305 propagation of deep-water waves from offshore of Monterey Bay to nearshore Santa Cruz, CA.

306 All model runs made use of a coarse, Monterey Bay grid. Its model resolution was $0.001^{\circ}$ in

307 latitude and longitude and it was run as a stationary model. Directional wave energy spectra 308 conditions were exported from the coarse resolution model and used as boundary conditions for 309 the nested, finer resolution model domain.

311 3.1.1 WECs larger than $15 \mathrm{~m}$

312 In addition to the coarse Monterey Bay grid, a smaller, nested model domain was employed in

313 SNL-SWAN for WEC devices greater than $15 \mathrm{~m}$ in diameter (see Figure 1). The finer, inner

314 nested model domain grid size (here, referred to as the Santa Cruz domain) was set equal to the

315 size of the particular WEC device being modeled. This was to establish each device as equivalent 316 to a model grid cell, which facilitates model performance evaluation and assessment.

$318 \quad 3.1 .2$ WECs smaller than $15 \mathrm{~m}$ 
319 WEC devices smaller than $15 \mathrm{~m}$ in diameter were modeled with a triple-nested SNL-SWAN

320 model. The reason for this additional nested grid was due to model allocation limitations when

321 attempting to model grid cells less than $15 \mathrm{~m}$ in dimension. The two finer resolution grids,

322 additional to the Monterey Bay domain, were the WEC model domain and Santa Cruz model

323 domain (Figure 1). For WECs with diameters less than $15 \mathrm{~m}$, directional wave energy spectra

324 conditions were exported from the Monterey Bay model and used as boundary conditions for the 325 WEC model domain.

327 The grid resolution of the nested WEC model domain was equal to the modeled WEC device 328 diameter. WEC devices that were $5 \mathrm{~m}$ in diameter required a smaller WEC domain due to model 329 computational limitations. WEC devices between $6 \mathrm{~m}$ and $10 \mathrm{~m}$ required a larger WEC domain 330 such that the entire WEC array of up to 100 devices would be accommodated. The WEC nested

331 grid model was also implemented as a stationary model. The WEC model wave spectrum

332 boundary conditions were applied along the southern offshore boundary of the Santa Cruz model

333 domain. The boundary between the WEC domain and the Santa Cruz domain was extended

334 sufficiently to the west in order to avoid boundary effects (e.g., shadowing) that may have

335 inaccurate model artifacts at the 18 model output locations.

337 The grid resolution of the innermost Santa Cruz model domain computational grid for SNL-

338 SWAN model runs with WEC smaller than $15 \mathrm{~m}$ in diameter was $0.00025^{\circ}$ in latitude and

339 longitude. The innermost Santa Cruz domain was also implemented as a stationary model. 
343 SNL-SWAN model sensitivity analysis results were retained for each model run. Results

344 included propagated wave heights, wave periods, wave directions, and near-bottom orbital

345 velocities at all grid points in the model domains. Further, the same wave properties were

346 extracted at each of the 18 distinct model output locations (Figure 1) to facilitate point-to-point

347 comparisons. Note that the primary focus of this study is the alteration of the incident wave

348 spectra downstream of the WEC, at nearshore locations. Any secondary waves potentially

349 generated and/or radiated by a WEC is assumed to be insignificant at the scales considered here.

350 Surface-to-surface evaluations (Figures 4, 5, and 6) compare the modeled scenario results to the

351 baseline scenario results, where the baseline scenario does not include WEC devices. Black

352 shading in Figures 4, 5, and 6 indicates no changes in wave parameter values from the baseline

353 scenario. Color bars are included in each figure to define the amount of change, where change is

354 defined as a percentage change from the baseline scenario, where:

$$
\text { Percentage Change }=\frac{(\text { Baseline-FinalValue })}{\text { Baseline }} \times 100
$$
or, for example,

$$
H_{S} \text { diff }=\frac{\left(\mathrm{H}_{\mathrm{S}} \text { Baseline }-\mathrm{H}_{\mathrm{S}} \mathrm{WEC}\right)}{H_{S} \text { Baseline }} \times 100
$$

359 Therefore, a positive change indicates a decrease in the value of the wave parameter in the

360 presence of a WEC array. Negative changes in MWD indicate clockwise rotation of wave

361 direction and positive changes indicate counter-clockwise rotation. 


\section{3.2.1 Significant Wave Height}

364 Results of significant wave height predictions from the sensitivity analysis for 50 Bref-SHB type

365 WECs using switches 1, 2, 3, and 4 are shown in Figure 4. The Bref-SHB WEC is relatively

366 small (Table 3) with relatively low power absorption values (Figure 3). Similar to the SNL-

367 SWAN model evaluation results, switch 2 and switch 4 resulted in greater wave height reduction

368 in the presence of the WEC array as compared to switch 1 and switch 3, again likely due to the

369 effects of the interpolating data when computing the RCW for switches 2 and 4 . Further

370 differences in decreased wave height are found when comparing results from employing constant

371 frequency versus frequency-dependent transmission coefficients (i.e. switch 1 and 2 versus

372 switch 3 and 4). For the Bref-SHB WEC, SNL-SWAN model runs with frequency-dependent

373 transmission coefficient (switches 3 and 4) result in up to $0.17 \%$ more wave height reduction

374 (output location number 7 comparison between switch 2 and switch 4). Increased wave height

375 reduction (or more power absorbed) for SNL-SWAN switch 3 and 4 runs is also observed for the

376 B-HBA, F3 OF, and F-HBA WEC device types regardless of WEC spacing or the number of

377 WECs in the array (not shown).

379 However, SNL-SWAN model runs with frequency-dependent transmission coefficient at times

380 resulted in less wave height reduction. This is illustrated in Figure 5 for the B-OF WEC type and

381 also holds true for the F-2HB and F-OWC devices. These three device types are the three largest

382 modeled WECs (Table 3) and have more or less symmetrical power matrices as a function of

383 period as compared to the smaller WECs, which generally exhibit maximum power at the lower

384 wave periods and decreasing power with increasing period (Figure 3). For the simulations 
performed here, smaller WECs (less than $10 \mathrm{~m}$ diameter) with asymmetric power matrices result

386 in more power absorption when employing frequency-dependent transmission coefficient. On the

387 other hand, model runs for larger (greater than $10 \mathrm{~m}$ diameter) WECs with more symmetrical

388 power matrices result in less simulated wave height reduction when modeled with frequency-

389 dependent transmission coefficient.

391 Switch 1 model results for all eight WEC buoy types are shown in Figure 6. The model

392 sensitivity parameters are 50 WECs with six diameter spacing for each of the WEC device types.

393 General spatial patterns in wave height reduction are similar for the other three switches and

394 other sensitivity parameters and thus are not shown. For all eight device types, the largest wave

395 height decreases were directly in the lee of the WEC arrays at output location numbers $7,8,11$,

396 and 12. In general, smaller devices had less impact on wave height as compared to larger buoy

397 sizes. Exceptions to this statement were the Bref-SHB buoy $(7 \mathrm{~m})$, which exhibited wave height

398 reductions of roughly equal magnitude as the B-HBA WEC type (5 m) and the F-OWC buoy (50

$399 \mathrm{~m}$ ), which had less of an impact on wave height than the $26 \mathrm{~m} \mathrm{B-OF} \mathrm{device} \mathrm{type} \mathrm{(Figure} \mathrm{6).} \mathrm{The}$

400 magnitude of wave height reduction was directly correlated to the WEC's power matrix values at

401 the modeled incoming wave height and period, with larger values resulting in more reduction in

402 wave height and vice versa.

403

404 The largest spatial (horizontal or along-shore) wave reduction effects were observed with the F-

405 OWC device, which makes intuitive sense given that these were the largest of the modeled

406 devices and were thus also spaced furthest apart, i.e. influencing a wider swath of the incident

407 wave field. 
409 Figure 7 summarizes model sensitivity to the following varied parameters: WEC buoy type,

410 WEC power absorption, number of WEC devices in the array and number of WECs in an array

411 including consideration of the sum total of power absorbed, and WEC spacing and WEC spacing

412 including the influence of the WEC array footprint area (i.e. wave height difference with and

413 without WECs divided by the total area occupied by a WEC array including the spacing in

414 between the WECs). General patterns observed in model sensitivity analysis are similar

415 throughout all four switches; therefore results are shown for a randomly selected switch.

416 Variability in wave height percent differences was largest for buoy type, i.e. the model was most

417 sensitive to WEC device type and WEC size with the exception of the F-OWC buoy. The power

418 matrix associated with each WEC was generally scaled to WEC size, i.e. larger buoys exhibited

419 greater power absorption as compared to smaller devices, with the exception of the F-OWC

420 buoy. WECs with higher power absorption resulted in greater wave height reduction. This is

421 illustrated in Figure 7B, where each WEC device type is represented by its average power

422 absorption as calculated by SNL-SWAN.

423

424 As expected, the larger the number of WECs in the array, the greater the difference between

425 modeled wave height with and without obstacles (Figure 7C). Again, these results are directly

426 correlated to WEC power absorption, i.e. more buoys will absorb more power. Model results are

427 insensitive to the number of WECs in an array when the total power absorption is considered.

428 Figure 7D shows wave height reduction divided by the sum total of SNL-SWAN calculated

429 absorbed power for all WECs in the array.

430 
431 Wave height reduction was relatively insensitive to WEC spacing (Figure 7E). However, when

432 the area of the WEC array footprint is considered, the model results vary with WEC spacing;

433 wave height reductions decrease with increasing WEC spacing (Figure 7F). This indicates that

434 closely spaced arrays have potentially more effects on nearshore wave propagation as compared

435 to arrays of WECs that are spaced farther apart. Furthermore, output locations that are directly in

436 the lee of the WEC array centerline (location numbers 7, 11, and 12) are more sensitive to the

437 spatial extent of the WEC array (Figure 8). The greater the horizontal distance, i.e. west and east

438 of the centerline of the lee of the WEC array, the more insensitive the model results are to WEC

439 array footprint area. Output location number 4 (Figure 8A) shows increased wave height

440 reduction with farther spaced WECs. This location is largely outside of the WEC array influence

441 except for simulations with the F-OWC WEC - the largest of all WECs modeled.

442

\section{$443 \quad 3.2 .2$ Near-Bottom Orbital Velocities}

444 Near-bottom orbital velocities (e.g. wave-driven currents) are directly proportional to surface

445 wave expression (i.e. significant wave height). Model simulated decreases in wave height due to

446 WEC power extraction cause a decrease in near-bottom orbital velocities, potentially altering the

447 ambient wave-driven currents in a nearshore environment. Consequently, the percentage

448 differences of the near-bottom orbital velocities were essentially equivalent to those computed

449 from the significant wave height model scenarios. Thus, near-bottom orbital velocity results are

450 not shown here. 
453 The percentage changes in peak wave periods during this study were negligible, as shown in

454 Figure 9. Within the model parameters, the frequency bin resolution was likely too large to

455 register small changes in wave periods (small changes in frequency would not cause a change in

456 frequency bin in model space). Note that for SNL-SWAN switch 3 and switch 4 simulations,

457 there was no observed changes in peak wave energy despite the frequency-dependent power 458 absorption, also indicating that the model frequency bin resolution was larger than any changes

459 in wave period.

461 3.2.4. Mean Wave Directions

462 Changes in mean wave directions are illustrated in Figure 10. Directional changes were most 463 sensitive to WEC spacing when the WEC array footprint area was considered. The closer spaced 464 arrays that occupied the smallest footprint resulted in larger directional rotations. However, all 465 percentage differences between the baseline scenario and a simulated WEC array translated to 466 within $\pm 4.5 \%$, corresponding to $\pm 9^{\circ}$ change in MWD. Negative changes indicated clockwise 467 rotation of wave direction. Positive changes indicated counter-clockwise rotation. Rotation, 468 when it occurred in the model, was relatively large because the directional bin spacing was equal 469 to $9^{\circ}$. Any changes less than this are indeterminable by the model as currently parameterized. 470 Zero wave direction change was observed for modeled devices smaller than $8 \mathrm{~m}$. It is thus 471 surmised that direction changes, if any, caused by the WEC devices were less than $9^{\circ}$. Higher 472 resolution changes in MWD could be ascertained with increased model directional resolution. 473 However, due to model allocation limitations in the present model configuration, model grid 474 resolution or model domain size would be compromised. 


\section{4. Summary and Conclusions}

478 The presence of WEC arrays have the potential to alter wave propagation patterns significantly

479 and affect coastal circulation patterns, sediment transport patterns, and alter ecosystem processes.

480 To help accelerate deployment of environmentally friendly WEC arrays, predictive modeling

481 tools have been developed to represent WEC induced changes in wave propagation and evaluate

482 the potential for environmental impact. The present study utilized a modified version (SNL-

483 SWAN) of an industry standard wave modeling tool, SWAN, to examine potential WEC array

484 deployment scenarios at a site on the California coast and investigate model sensitivity so that

485 the model can be effectively and confidently used in environmental studies. This analysis built

486 upon a previous sensitivity analysis in which SWAN model parameters were varied to examine

487 their effect on model results (Chang et al., 2014).

489 In the present study, SNL- SWAN, was evaluated against the native SWAN code and used to 490 investigate the effects of different WEC devices on nearshore wave propagation. SNL-SWAN 491 sensitivity analysis studies were performed to examine the effects of WEC variations (WEC

492 device type and size, number of WEC devices in an array, and the spacing of the WEC devices 493 within the array) on near-field and far-field wave conditions in the lee of the WEC devices.

495 The results illustrate that, given the present model setup, the wave heights and associated near-

496 bottom orbital velocities showed decreases of up to $30 \%$ between baseline and modeled

497 conditions for 100 devices of the B-OF buoy type. The B-OF power matrix values were largest 
498 for an incident wave height of $1.7 \mathrm{~m}$. Other buoy types resulted in less than $15 \%$ differences in

499 modeled wave height with and without obstacles, with lesser influence for buoys less than $10 \mathrm{~m}$

500 in diameter. Although the F-OWC device was the largest device modeled, its power matrix

501 values for an incident wave height of $1.7 \mathrm{~m}$ were less than that of the B-OF device and hence its

502 wave reduction potential was less. However, the F-OWC effects extended over a larger spatial

503 extent due to its size and spacing, thereby potentially having a greater effects on the local

504 shoreline.

505

506 Model output locations located to the west (output locations 1 through 6) showed relatively little

507 to no change in wave heights compared to the baseline scenario. The largest wave height

508 differences were observed downstream of the array near the array centerline (output locations 7

509 through 12), where the largest wave shadowing effects are predicted. Additional model output

510 locations to the east of the array (output locations 13 through 18) indicated relatively small

511 changes in wave heights for buoys larger than $9 \mathrm{~m}$ in diameter. This is intuitive given that the

512 modeled incident wave direction was from the southwest and these waves refracted toward the

513 shoreline in a counter-clockwise manner.

515 Wave directions and periods did not appear to be sensitive to changes in WEC and WEC array

516 characteristics except when considering WEC array footprint sizes. However, additional analysis

517 is required to fully explore the model sensitivity of peak wave period and mean wave direction to

518 the varying of the WEC parameters.

519 
520 The SNL-SWAN Version 1.0 source code is publically available (https://github.com/SNL-

521 WaterPower/SNL-SWAN/tree/master/bin), has been verified, and has undergone preliminary

522 validation by comparison to experimental wave tank data. However, it is important to utilize

523 ongoing laboratory studies and future field tests to determine the most appropriate

524 implementation of WEC power performance data. Until actual power matrix values become

525 available or further WEC-specific model enhancements are validated, this study shows that

526 environmental assessments of WEC devices should focus on evaluating a range of WEC

527 characteristics in order to determine the limits of the potential environmental effects resulting

528 from the presence of a WEC array. For more information about the ongoing development,

529 validation, and applications of SNL-SWAN, please see Porter et al. (2015) and Ruehl et al.

530 (2015).

532 In summary, the present study developed a baseline model understanding while investigating the 533 effects of a range of WEC devices. The sensitivity, optimization, and behavior of the model for

534 various WEC devices provided the basis for a solid model understanding giving the confidence 535 necessary for future WEC evaluations.

\section{Acknowledgments}

538 This research was made possible by support from the Department of Energy's Wind and Water

539 Power Technologies Office, funded by the U.S. Department of Energy. Sandia is a multi-

540 program laboratory operated by Sandia Corporation, a Lockheed Martin Company, for the

541 United States Department of Energy's National Nuclear Security Administration under contract 
542 DE-AC04-94AL85000. Special thanks to Ari Posner for early developmental efforts associated 543 with SNL-SWAN and to Jason Magalen for initial SWAN model set-up and sensitivity studies. 


\section{$544 \quad$ Figure Captions}

546 Figure 1. The Monterey Bay domain, WEC domain (bounded by solid lines), and Santa Cruz

547 (bounded on three sides - north, west, and east - by dashed lines) SNL-SWAN model domains

548 for devices between $6 \mathrm{~m}$ and $15 \mathrm{~m}$ in diameter. The inset shows a close-up view of the WEC and

549 Santa Cruz domain (boundary between the two marked by the solid line) with 18 output

550 locations and simulated WEC array illustrated.

552 Figure 2. Simulated wave height for model evaluation runs: (A) native SWAN with transmission 553 coefficient equal to 0.86 , and SNL-SWAN (B) switch 0 with transmission coefficient equal to

554 0.86, (C) switch 1, (D) switch 2, (E) switch 3, and (F) switch 4. The text on the left of each panel 555 indicates the simulated wave height at each of the 18 output locations, which are shown as black 556 dots in all panels and numbered in panel (A). The simulated WEC array is illustrated as small 557 black dots on the $40 \mathrm{~m}$ depth contour.

559 Figure 3. Power matrices as a function of significant wave height and peak wave period 560 computed for the eight different WEC device types modeled in SNL-SWAN sensitivity analysis 561 (from Babarit et al., 2012). (A) B-HBA, (B) B-OF, (C) Bref-HB, (D) Bref-SHB, (E) F-2HB, (F) 562 F3 OF, (G) F-HBA, and (H) F-OWC. Color bar scales differ amongst different WEC types.

564 Figure 4. Significant wave height percentage decrease using SNL-SWAN switch (A) 1, (B) 2, 565 (C) 3, and (D) 4 for model runs specifying 50 Bref-SHB type WECs with $6 \mathrm{~m}$ spacing on the 40 
$566 \mathrm{~m}$ depth contour. Percent decreases at each of the 18 output locations are listed on the left. The

567 device diameters represented in the figure are not to scale.

568

569 Figure 5. Significant wave height percentage decrease using SNL-SWAN switch (A) 1, (B) 2,

570 (C) 3, and (D) 4 for model runs specifying 50 B-OF type WECs with $6 \mathrm{~m}$ spacing on the $40 \mathrm{~m}$

571 depth contour. Percent decreases at each of the 18 output locations are listed on the left. The

572 device diameters represented in the figure are not to scale.

573

574 Figure 6. Significant wave height percentage decrease using SNL-SWAN with a simulated array 575 of 50 WECs spaced $6 \mathrm{~m}$ apart on the $40 \mathrm{~m}$ depth contour using switch 1 for (A) Bref-HB, (B) B576 HBA, (C) Bref-SHB, (D) F-HBA, (E) F3 OF, (F) F-2HB, (G) B-OF, and (H) F-OWC WEC

577 types. Percent differences at each of the 18 output locations are indicated on the left. Device 578 diameters are not to scale.

580 Figure 7. Wave height reduction as affected by variations in (A) WEC device type (and size), (B)

581 WEC device (and size) represented by each WEC's average power absorption, (C) number of 582 WECs in a WEC array, (D) number of WECs in an array considering the total absorbed power, 583 (E) WEC spacing, and (F) WEC spacing considering the WEC array footprint area. Results are 584 from SNL-SWAN switch 4 simulations.

586 Figure 8. Wave height reduction as affected by variations in WEC spacing considering the WEC 587 array footprint area for nine different output locations as indicated in each panel. See Figure 2 for 588 a map of output locations. Results are from SNL-SWAN switch 3 simulations. 
590 Figure 9. Peak wave period reduction as affected by variations in (A) WEC device type (and 591 size), (B) number of WECs in a WEC array, (C) WEC spacing, and (D) WEC spacing with 592 consideration of the WEC array footprint area. Results are from SNL-SWAN switch 3 593 simulations.

594

595 Figure 10. Mean wave direction as affected by variations in (A) WEC device type (and size), (B) 596 number of WECs in a WEC array, (C) WEC spacing, and (D) WEC spacing considering the 597 WEC array footprint area. Negative differences indicate clockwise rotation and positive 598 differences indicate counterclockwise rotation. Results are from SNL-SWAN switch 2 599 simulations. 
601

602 B-HBA Bottom-fixed heave-buoy array

603 B-OF Bottom-fixed oscillating flap

604 Bref-HB Small bottom-referenced heaving buoy

605 Bref-SHB Bottom-referenced submerged heave-buoy

606 F-2HB Floating two-body heaving converter

$607 \quad$ F3 OF Floating three-body oscillating flap device

608 F-HBA Floating heave-buoy array

609 F-OWC Floating oscillating water column

$610 \mathrm{H}_{\text {incident }} \quad$ Incident or incoming wave height

$611 \mathrm{H}_{\text {lee }} \quad$ Wave height in the lee of a simulated WEC or obstacle

$612 \mathrm{H}_{\mathrm{s}} \quad$ Significant wave height

$613 \quad \mathrm{~K}_{\mathrm{t}} \quad$ Transmission coefficient

614 ME Mean error or bias

615 MWD Mean wave direction

616 NDBC National Data Buoy Center

617 NOAA National Oceanic and Atmospheric Administration

618 Pabsorbed Wave power absorbed by a WEC

619 Pincident Incident or incoming wave power

$620 \quad P_{\text {lee }} \quad$ Wave power in the lee of a simulated WEC or obstacle

621 RCW Relative capture width

622 RMSE Root mean square error 
623 SI Scatter index

624 SNL Sandia National Laboratories

625 SWAN Simulating WAves Nearshore

626 SNL-SWAN Modified SWAN model

$627 \quad \mathrm{~T}_{\mathrm{p}} \quad$ Peak wave period

628 WEC Wave energy converter 
631 ASR Limited (2007) Review of Wave Hub Technical Studies: Impacts on inshore surfing 632 beaches, Hamilton, New Zealand.

634 Barbarit, A., Hals, J., Muliawan, M.J., Kurniawan, A., Moan, T., Krokstad J., 2012. Numerical

635 benchmarking study of a selection of wave energy converters. Renew. Energ. 41, 44-63.

Bento, A.R., Rusu, E., Martinho, P., Guesdes Soares, C., 2014. Assessment of the changes induced by a wave energy farm in the nearshore wave conditions. Comput. Geosci. 71, 50-61.

640 Booij, N., Ris, R.C., Holthuijsen, L.H., 1999. A third-generation wave model for coastal regions.

641 Part I: Model description and validation. J. Geophys. Res. 104, 7649-7666.

643 Chang, G, Jones, C., Hansen, D., Twardowski M., Barnard, A., 2010. Prediction of Optical

644 Variability in Dynamic Near-shore Environments: Task Completion Report \#3 - Numerical

645 Modeling and Verification, Sea Engineering, Inc., Santa Cruz, CA.

647 Chang, G., Magalen, J., Jones, C., Roberts J., 2014. Investigation of wave energy converter

648 effects on wave fields: A modeling sensitivity study in Monterey Bay, CA, Sandia Report, 649 SAND2014-16840, Sandia National Laboratories, Albuquerque, NM.

651 Collins, 1972. Prediction of shallow water spectra. J. Geophys. Res. 93 (C1), 491-508. 
653 Copping, A., Hanna, L., Van Cleve, B., Blake, K., Anderson, R., 2014. Environmental risk

654 evaluation system - An approach to ranking risk of ocean energy development on coastal and 655 estuarine environments. Est. Coast. 38, 287-302.

657 d'Angremond, K., van der Meer, J.W., de Jong, R.J., 1996. Wave transmission at low-crested 658 structures, Proc. $25^{\text {th }}$ Int. Conf. Coastal Engng., ASCE, Orlando, pp. 2418-2427.

660 Delft University of Technology, 1993. SWAN User Manual, SWAN Cycle III version 41.01, 661 Delft, The Netherlands.

662

663 Goda, Y., Takeda, H., Moriya, Y., 1967. Laboratory investigation of wave transmission over 664 breakwaters. Rep. Port and Harbour Res. Inst. 13.

665

666 Gonzalez-Santamaria, R., Zou, Q., Pan, S., 2013. Impacts of a wave farm on waves, currents, 667 and coastal morphology in south west England. Est. Coast. 1, 1-14.

668

669 Hagerman, G., Bedard, R., 2003. Guidelines for Preliminary Estimation of Power Production by 670 Offshore Wave Energy Conversion Devices, Technical Report E2I EPRI-WP-US-001, 671 Electricity Innovation Institute, Electric Power Research Institute, Palo Alto, CA, USA.

673 Iglesias, G., Carballo, R., 2014. Wave farm impact: The role of farm-to-coast distance. Renew. 674 Energ. 69, 375-385. 
676 Leeney, R., Greaves, D., Conley, D., O’Hagan, A., 2014. Environmental impact assessments for

677 wave energy developments - Learning from existing activities and information future research

678 priorities. Ocean Coast. Manage. 99, 14-22.

679

680 Millar, D.L., Smith, H.C.M., Reeve, D.E., 2007. Modelling analysis of the sensitivity of

681 shoreline change to a wave farm. Ocean Eng. 34, 884-901.

682

683 Palha, A., Mendes, L., Fortes, C.J., Brito-Melo, A., Sarmento, A., 2010. The impact of wave

684 energy farms in the shoreline wave climate: Portuguese pilot zone case study using Pelamis

685 energy wave devices. Renew. Energ., 35, 62-77.

686

687 Porter, A., Ruehl, K., Chartrand, C., 2014. Further development of SNL-SWAN, a validated

688 wave energy converter array modeling tool, in: Proc. $2^{\text {nd }}$ Marine Energy Technology

689 Symposium, Seattle, WA, pp. 1-9.

690

691 Porter, A., Ruehl, K., Chartrand, C., and Smith, H., 2015. Development and Release of the Open-

692 Source Wave Climate Environment Assessment Tool SNL-SWAN. In: Proceedings of the 3rd

693 Marine Energy Technology Symposium, Washington D.C. USA.

694

695 Ris, R.C., Booij, N., Holthuijsen, L.H., 1999. A third-generation wave model for coastal regions.

696 Part II: Verification. J. Geophys. Res. 104, 7667-7682.

697 
698 Ruehl, K., Chartrand, C., Porter, A., 2014. SNL-SWAN Manual, SAND Report, Sandia National

699 Laboratories, Albuquerque, NM.

700

701 Ruehl, K., Porter, A., Chartrand, C., Smith, H., Chang, G., and Roberts, J., 2015. Development,

702 Verification and Application of the SNL-SWAN Open Source Wave Farm Code. In: Proceedings

703 of the 11th European Wave and Tidal Energy Conference, Nantes, France.

704

705 Ruehl, K., Porter, A., Posner, A., Roberts, J., 2013. Development of SNL-SWAN, a Validated

706 Wave Energy Converter Array Modeling Tool, presented at the EWTEC 2013, Denmark.

707

708 Rusu, E., Guedes Soares, C., 2008. Wave energy assessments in the coastal environment of

709 Portugal continental. In: Proceedings of the $27^{\text {th }}$ International Conference on Offshore

710 Mechanics and Arctic Engineering (OMAE 2008) Estoril, Portugal, ASME, OMAE2008-57820.

711

712 Rusu, E., Guedes Soares, C., 2009. Numerical modeling to estimate the spatial distribution of the

713 wave energy in the Portuguese nearshore. Renew. Energ. 34 (6), 1501-1516.

714

715 Rusu, E., Guedes Soares, C., 2013. Coastal impact induced by a Pelamis wave farm operating in

716 the Portuguese nearshore. Rewew. Energ. 58, 34-49.

717

718 Smith, H.C.M., Pearse, C., Millar, D.L., 2012. Further analysis of change in nearshore wave

719 climate due to an offshore wave farm: An enhanced case study for the Wave Hub site. Renew.

720 Energ. 40, 51-64. 
722 Tiron, R., Mallon, R., Frederic, D., Reynaud, E.G., 2015. The challenging life of wave energy

723 devices at sea: A few points to consider. Renew. Sust. Energ. Rev. 43, 1263-1271.

724

725 TOMAWAC - Wave propagation in coastal areas. [Online]. Available:

726 http://www.opentelemac.org/index.php/presentation?id=20. [Accessed: 27-Jan-2014]. 
Figure 1

Longitude $\left({ }^{\circ} \mathrm{W}\right)$

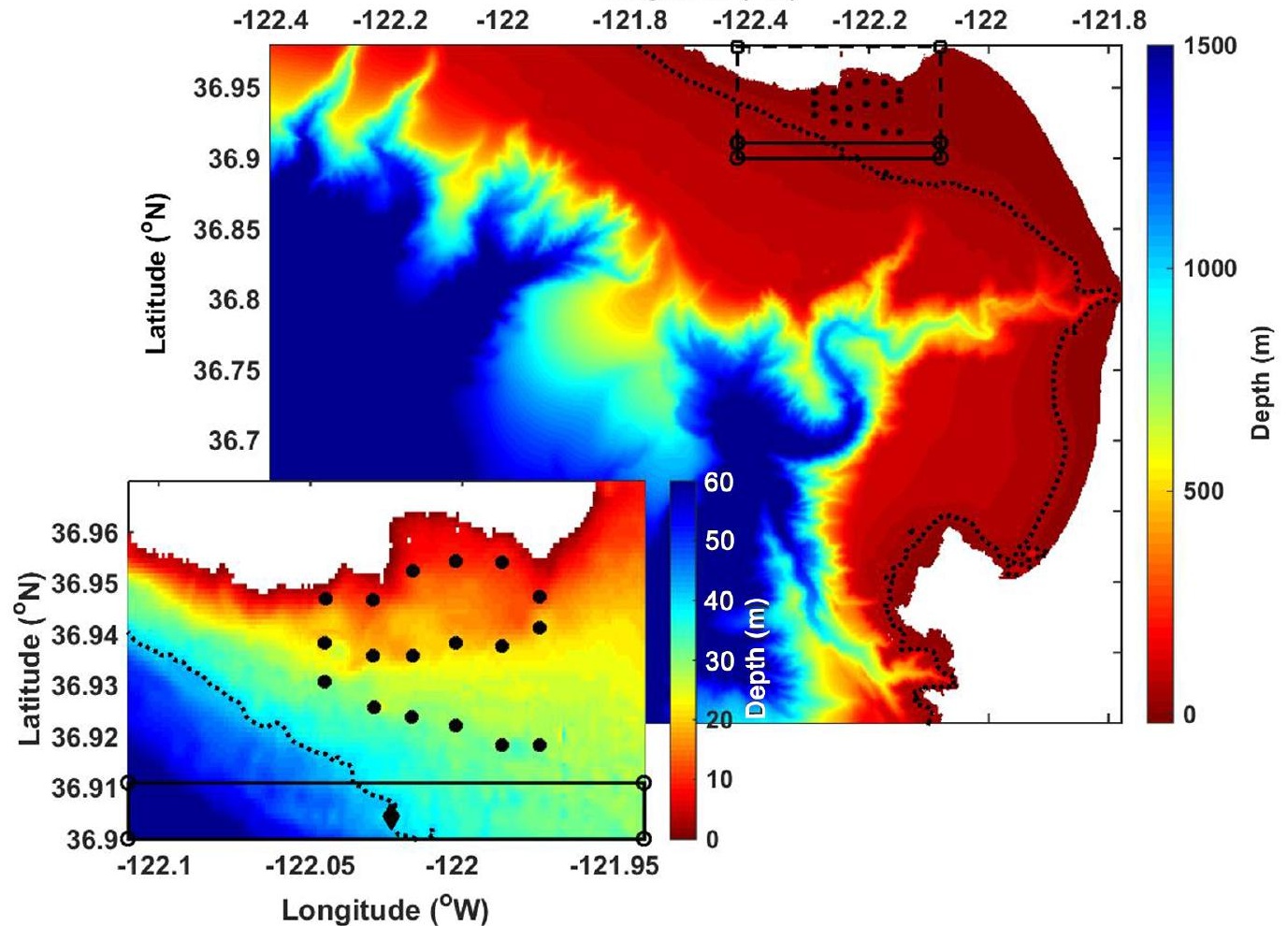



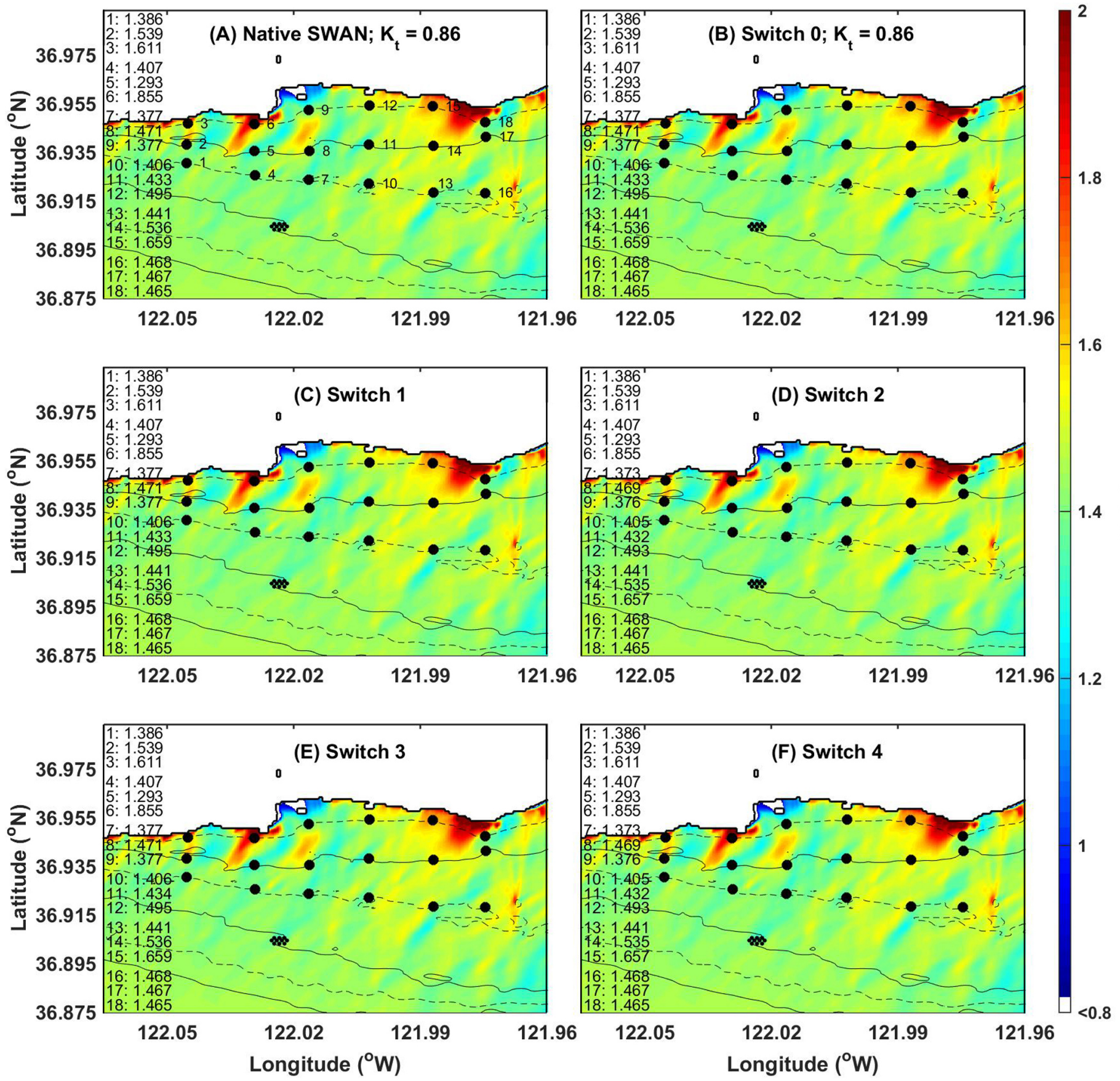
Figure 3
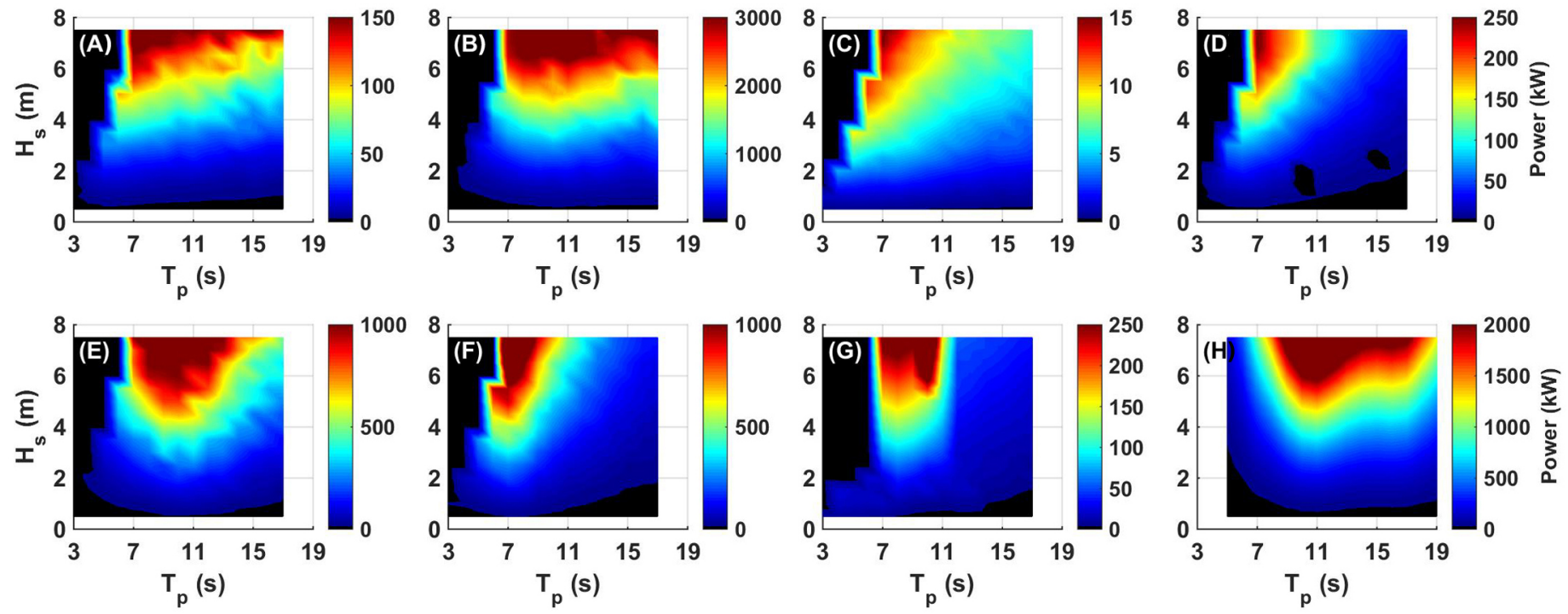

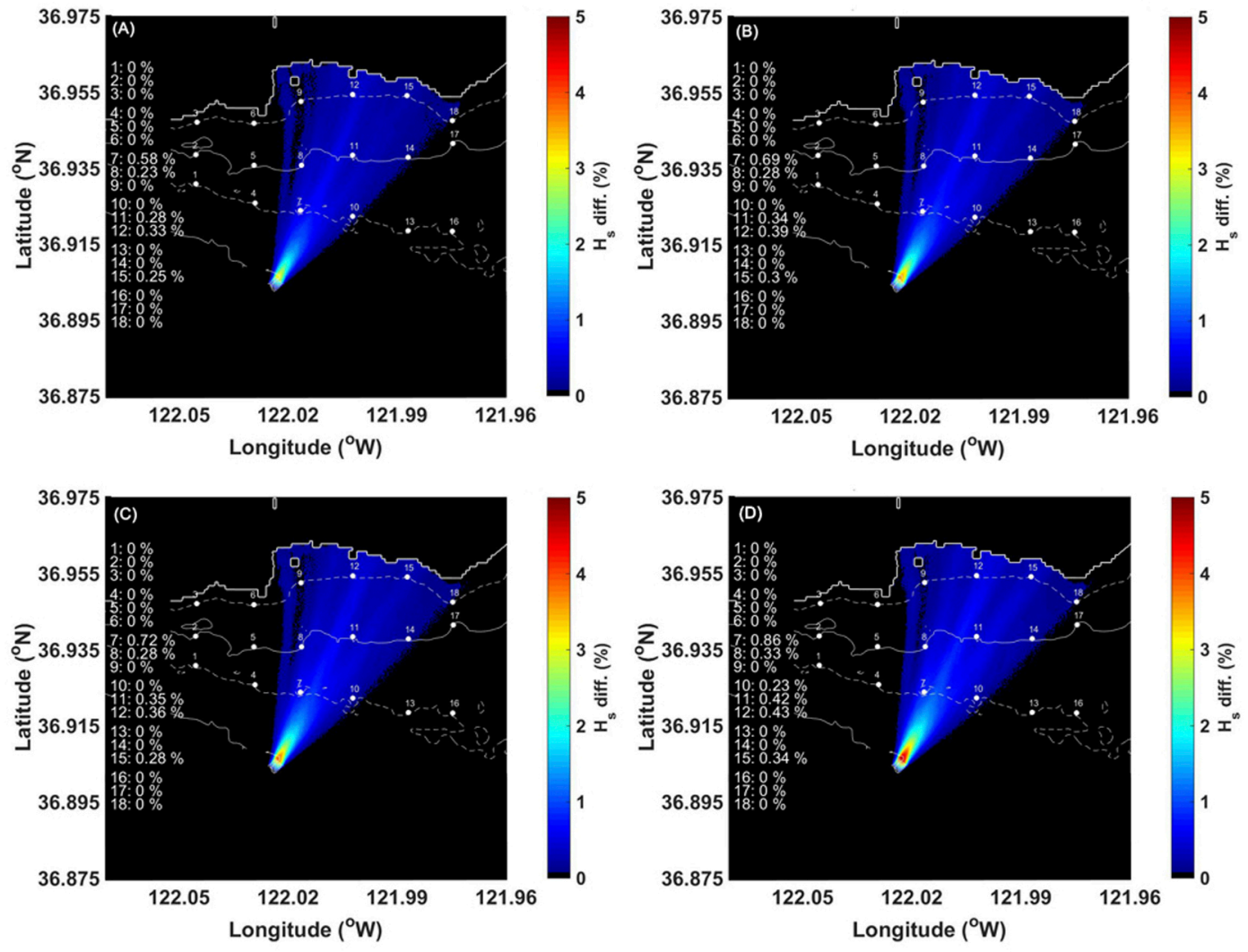

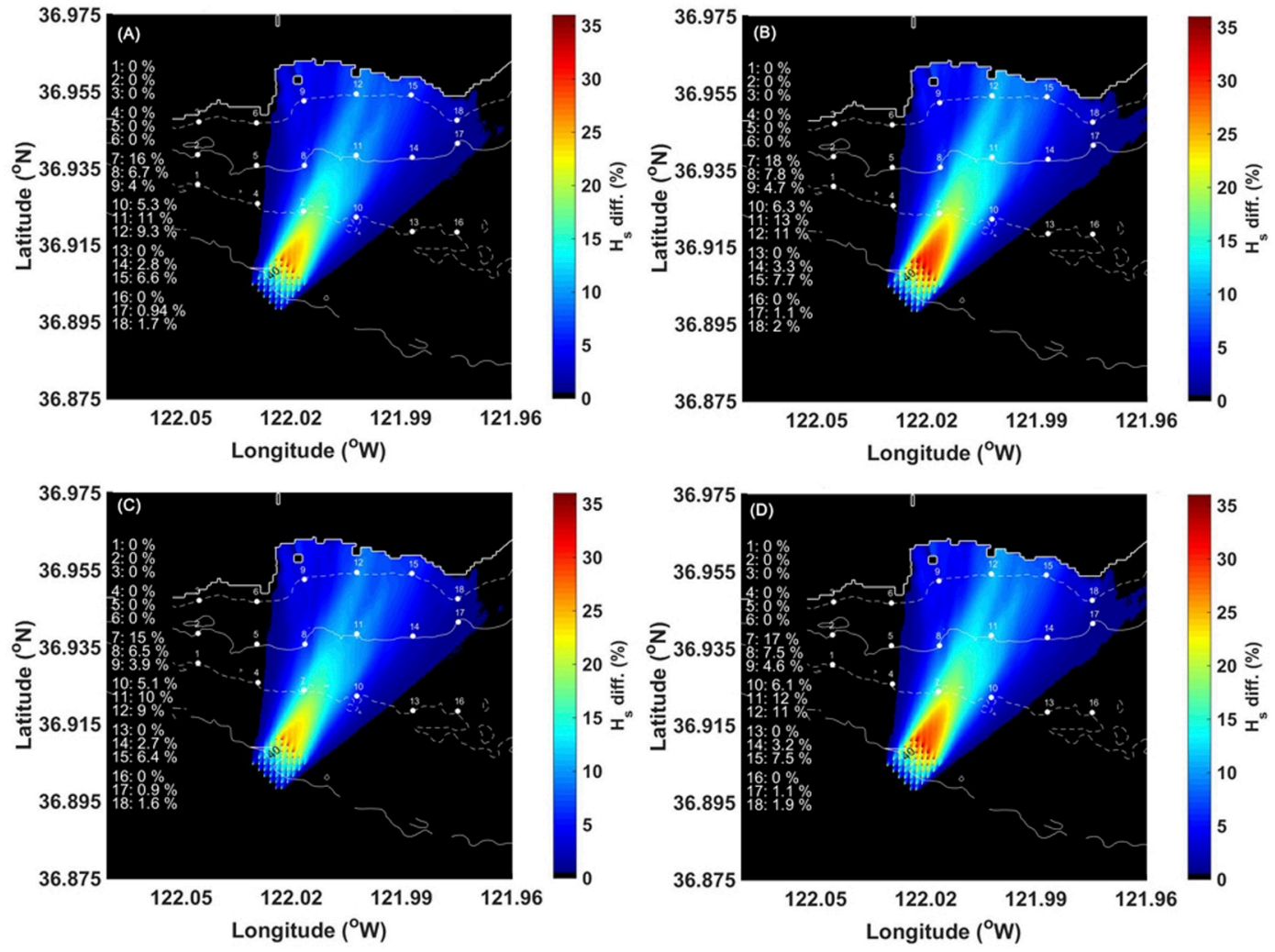

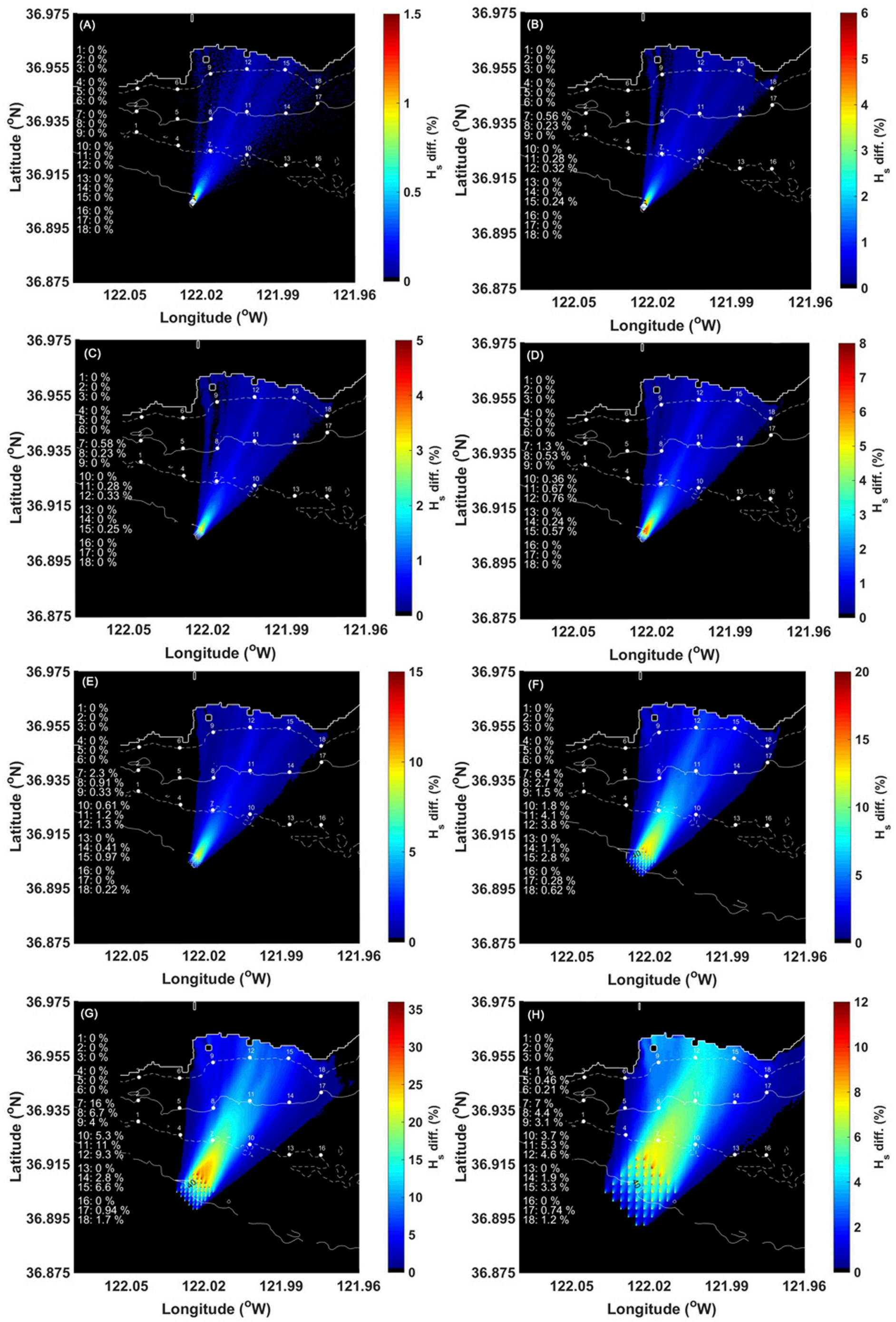

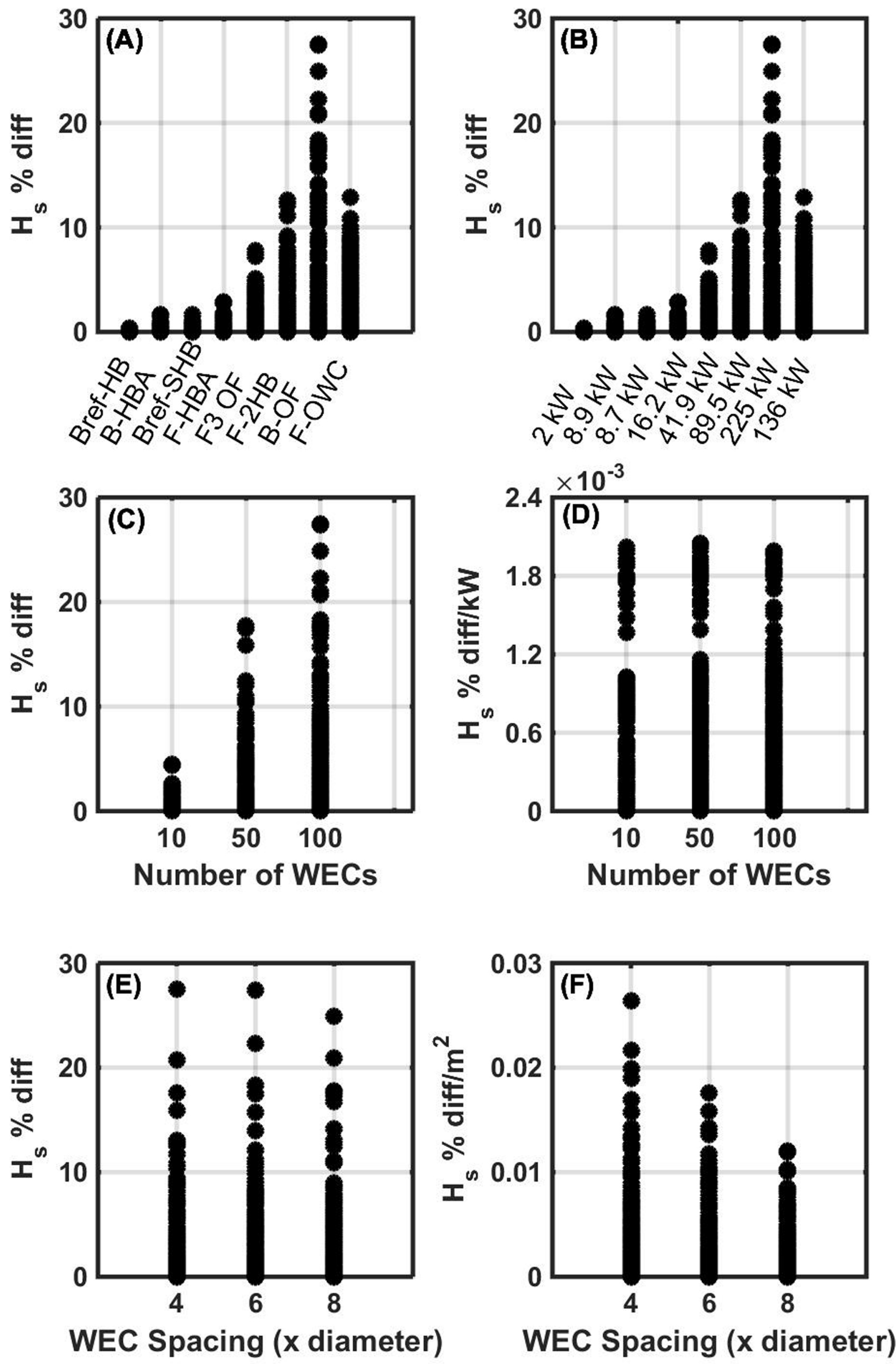

WEC Spacing (x diameter) 

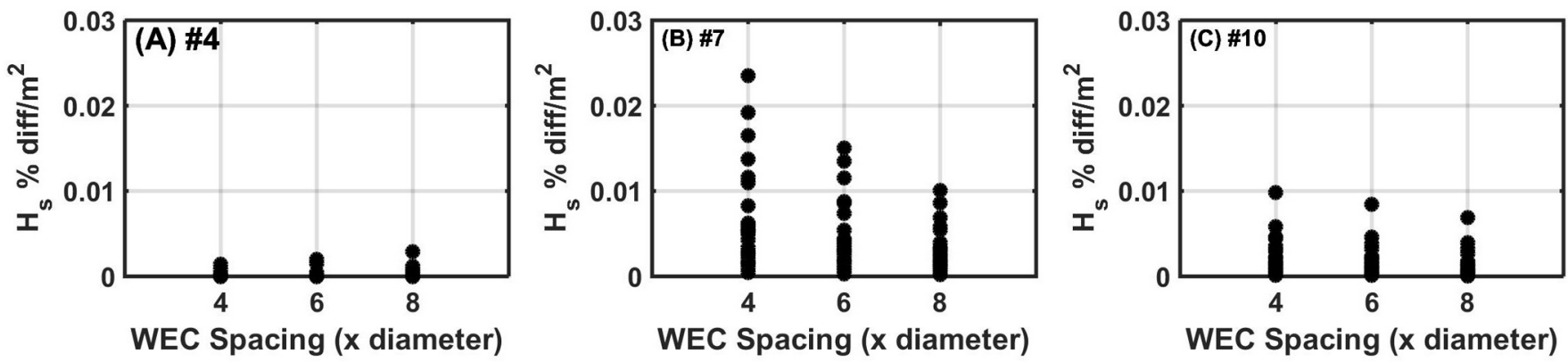

WEC Spacing (x diameter)
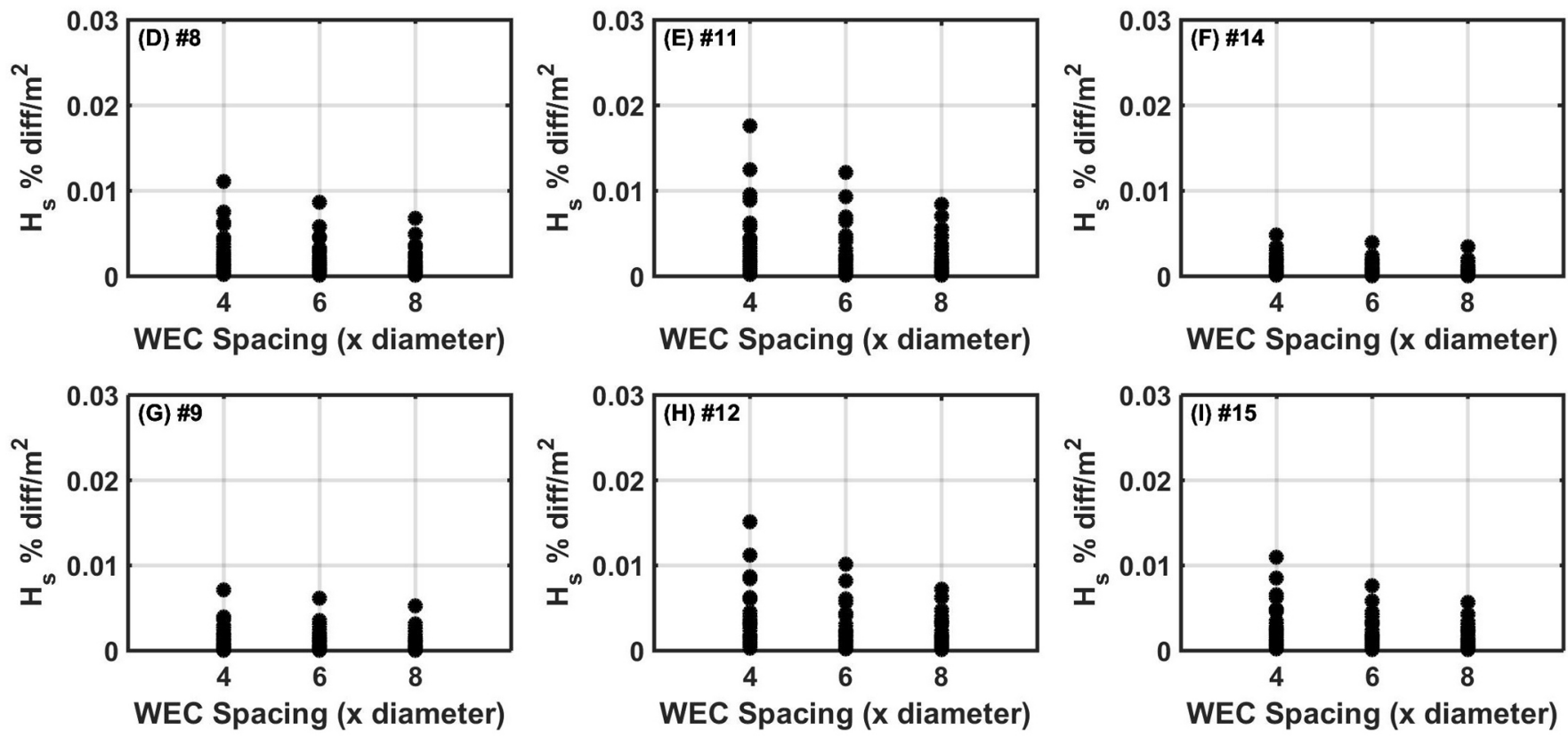

WEC Spacing (x diameter) 
Figure 9
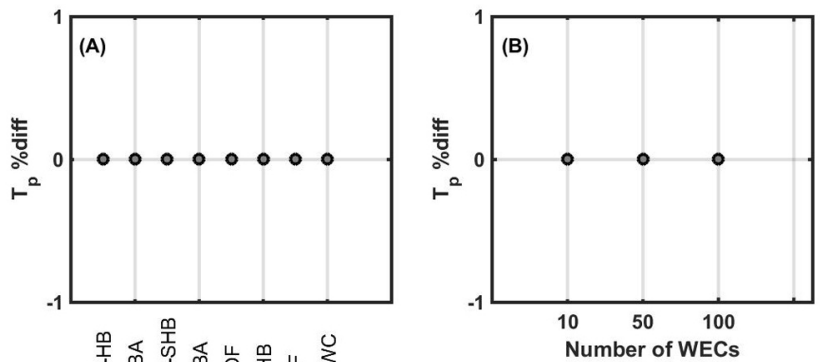

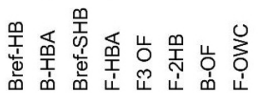
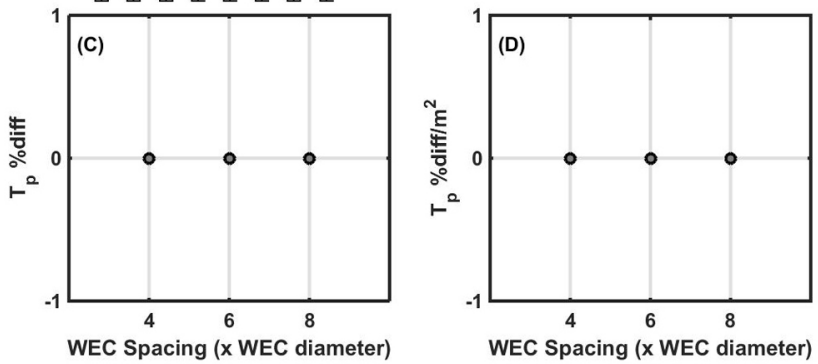

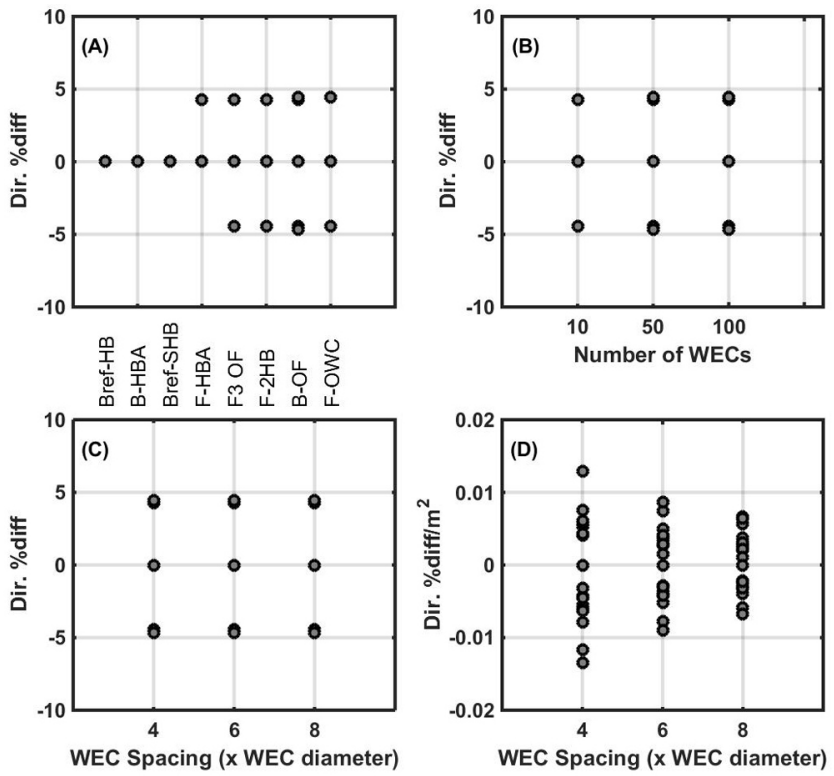